\title{
Usuario mapuche: significado hipertensión arterial, Temuco, 2016
}

\author{
Mapuche User: Meaning of Arterial Hypertension, Temuco, 2016 \\ Usuário Mapuche: significado hipertensão arterial, Temuco, 2016
}

Pamela Chávez-Coña'1, Mónica Illesca-Pretty², Mirtha Cabezas-González³, Gloria Muñoz-Navarro ${ }^{4}$

1 Enfermera, magíster en Enfermería. Académica Escuela de Enfermería. Universidad Autónoma de Chile, Temuco, Chile. pame.chavez.c@gmail.com.

2 Enfermera, Dra. en Salud. Académica Departamento Medicina Interna. Oficina de Educación en Ciencias de la Salud. Facultad de Medicina, Universidad de La Frontera.Temuco, Chile. monica.illesca@ufrontera.cl

3 Químico farmacéutico, diplomada en Educación. Académico Departamento Ciencias Preclínicas, Oficina de Educación en Ciencias de la Salud, Facultad de Medicina, Universidad de La Frontera. Temuco, Chile. mirtha. cabezas@ufrontera.cl

4 Nutricionista, Dra. en Ciencias Médicas. Académica Escuela de Nutrición. Universidad Autónoma de Chile, Temuco, Chile.gloria.mn@gmail.com

Recibido: 15/04/2017. Aprobado: 17/05/2018. Publicado: 30/05/2018

Chávez-Coña P, Illesca-Pretty M, Cabezas-González M; Muñoz-Navarro G. Usuario mapuche: significado hipertensión arterial, Temuco, 2016. Rev. Fac. Nac. Salud Pública. 2018;36(2):74-84. Dor:10.17533/udea.rfnsp.v36n2a10

\section{Resumen}

Objetivo: Conocer el significado que otorga el usuario mapuche, adscrito al Programa Salud Cardiovascular 2016, Consultorio Makewe, Temuco, Chile, a la hipertensión arterial. Metdología: Investigación bajo el paradigma cualitativo, con base en la teoría fundamentada. La muestra fue conformada por veintidós usuarios, no probabilística, intencionada de casos por criterios y conveniencia, de acuerdo con criterios de inclusión. La recolección de información se hizo mediante dos grupos focales y ocho entrevistas en profundidad, hasta llegar al punto de saturación, previa firma de consentimiento informado. El análisis se caracterizó por ser dinámico y creati- vo, basado en la estrategia de método comparativo constante, realizando codificación y análisis simultáneo. La validez se comprobó con criterios de rigor y fue triangulada por investigadores. La investigación fue autorizada por el Comité Ético Científico de la Universidad de La Frontera. Resultados: Para hipertensión arterial emergieron cuatro categorías: "Significado" (definición del concepto), "Causa” (percepción), "Convivir" (cambios) y "Tratamiento" (farmacológico, herbolaria, machi — curandera o consejera-). Conclusiones: Sin haber definido la patología, los mapuches la asocian a signos, síntomas y causas; confían en tratamientos otorgados por un equipo 
de salud, pero no en las/los machis, reconociendo algunas de ellas como buenas, las que por alta demanda y costo son inaccesibles. En general, se ha descuidado la Ley Indígena 19253 de 1993, que da lugar a la institucionalización del Programa de Salud con Población Mapuche (PROMAP), dependiente del Servicio de Salud Araucanía.
--------Palabras clave: hipertensión, salud de poblaciones indígenas, pueblo mapuche, Ley Indígena 19253 de 1993, enfermedades cardiovasculares, atención de salud, servicios de salud.

\section{Abstract}

Objective: to understand the meaning that the Mapuche user subscribed to the Programa Salud Cardiovascular 2016, Makewe Clinic, Temuco, Chile assigns to arterial hypertension. Methodology: the research was performed under the qualitative paradigm, based on grounded theory. The nonprobabilistic sample was made up of twenty-two users. The cases were chosen by convenience, following inclusion criteria. The information was collected through focal groups and eight in-depth interviews, until saturation happened, and all participants were required to sign consent forms. Validity was ratified using standard criteria and researchers triangulated the data. The Ethics and Scientific Committee of Universidad de La Frontera authorized the study. Results: four categories for arterial hypertension appeared: "Meaning" (concept's definition), "Cause" (perception), "Living with the condition" (chan- ges) and "Treatment" (pharmacologic, herbal, Machi- healer or counselor-). Conclusions: without defining the pathology, the Mapuche associate the signs, symptoms and causes to it. They trust the treatment indicated by a medical team, but not the one prescribed by Machis. Although they recognize some of these Machis are good, their high demand or high cost makes them unattainable. In general, the Ley Indigena [Indigenous Peoples' Act] 19253 of 1993 has been neglected, even if it allows the institutionalization of the Programa de Salud con Población Mapuche (Health Care Program for Mapuche people- PROMAP in Spanish), a population that depends on the health care service provided by the Araucanía Region.

Key words: hypertension, health of indigenous peoples, Mapuche people, Indigenous Peoples' Act 19253, cardiovascular disease, health care, health services.

\section{Resumo}

Objetivo: conhecer o significado que dá o usuário Mapuche adscrito ao Programa Salud Cardiovascular 2016, consultório Makewe, Temuco, Chile, à hipertensão arterial. Metodologia: a pesquisa foi feita sob o paradigma qualitativo, baseada na teoria fundamentada. A amostra teve vinte e dois usuários, não probabilística, com intenção de casos por critério e conveniência, segundo os critérios de inclusão. A informação foi coletada mediante grupos focais e oito entrevistas aprofundadas, até atingir saturação, depois de ter assinado o consentimento. A análise caracterizou-se por ser dinâmica e criativa, baseada na estratégia de metodologia comparativa constante, realizando codificação e análise simultâneo. Comprovou-se a validez utilizando critérios correspondentes e com triangulação de pesquisadores. A pesquisa foi autorizada pelo Comité Ético e Científico da Universidad de La Frontera. Resultados: sur-

\section{Introducción}

Chile se constituye como un espacio pluriétnico, marcado por la coexistencia de distintos grupos originarios, caracterizándose cada uno por su visión de entender y actuar en el mundo, situación que en el ámbito del proceso salud-enfermedad se manifiesta por la coexistencia de las formas que lo abordan. De esta manera, cuando se está frente a un determinado grupo humano, se pueden encontrar creencias, conocimientos, percep- giram quatro categorias para hipertensão arterial: "Significado" (definição do conceito), "Causa" (percepção), "Conviver" (mudanças) e "Tratamento" (farmacológico, herbolária, Machi -curandeira ou aconselhadora-). Conclusões: sem ter definido a patologia, os Mapuches a associam aos sinais, sintomas e causas. Eles confiam nos tratamentos fornecidos por uma equipe de saúde, mas não nas/nos Machis, reconhecendo algumas delas como boas, as que por alta demanda e custos não são acessíveis. Em geral, tem se descuidado a Ley Indígena 19253 de 1993, que permite a institucionalização do Programa de Salud con Población Mapuche (PROMAP), dependente do Servicio de Salud Araucanía.

-Palavras-chave: hipertensão, saúde de populações indígenas, povo Mapuche, lei indígena 19253, doenças cardiovasculares, atendimento de saúde, serviços de saúde. ciones y prácticas terapéuticas validadas socialmente por quienes la sustentan [1].

Debido a esto, desde la década de los noventa del siglo $\mathrm{xx}$, a nivel ministerial se han tomado numerosas decisiones, desarrollado estrategias, planes y programas en materia de salud para los pobladores originarios [2]. Antes de 1993, las únicas políticas que se llevaron a cabo, en el marco del indigenismo, fueron de integración o asimilación al Estado nación chileno. En 1993 se sanciona la Ley Indígena 19253, que da lugar 
a la institucionalización del Programa de Salud con Población Mapuche (PROMAP) dependiente del Servicio de Salud Araucanía, cuyo propósito es "contribuir a la disminución de brechas de inequidad, en la situación de salud de los pueblos indígenas, a través de la construcción participativa de planes de salud que reconozcan la diversidad cultural, promuevan la complementariedad entre sistemas médicos y provean servicios de salud adecuados que respondan a necesidades, derechos y perfiles epidemiológicos específicos" [3].

Actualmente, se dispone de normativas específicas, como la Norma General Administrativa N. ${ }^{\circ}$ 16, sobre "Interculturalidad en los Servicios de Salud", que en uno de sus artículos señala: "en la formulación de los Planes de Salud Pública regionales, la Autoridad Sanitaria Regional velará por la incorporación de estrategias y actividades destinadas a identificar, monitorear y en lo posible a disminuir las brechas de equidad en la situación de salud y medio ambiente de la población indígena, y proponer las medidas intersectoriales pertinentes" [4].

Con base en Davidson et al., Hasen define la interculturalidad como "una relación entre culturas dinámicas, en la cual existe necesariamente reciprocidad, voluntad y horizontalidad, reconociendo que hay puntos de encuentro donde se pueden negociar y otros donde se mantienen las especificidades, respetando las diferencias, mejorando la salud, ganándose espacios desde los pueblos originarios y cediéndolos desde las instituciones oficiales" [1].

Al respecto, la región de La Araucanía, ubicada al centro de Chile, ha sido pionera en el intento de incorporar la cosmovisión de salud y enfermedad de la sociedad mapuche en las prácticas de atención del equipo multidisciplinario [5]; sin embargo, este proceso de cambio cultural ha demostrado ser gradual y heterogéneo, presentándose desde "negaciones" y "aceptaciones", hasta, en algunos casos, reconocer "otros liderazgos" en salud, comenzando por construir relaciones de confianza entre ambos sistemas médicos y modelos sanitarios. Para la medicina oficial, significa pasar de un modelo biomédico a uno más integrador, con un enfoque psicosociocultural, espiritual y biológico, en el cual se debe reconocer que existen otros actores, los agentes de salud indígena, que también tienen conocimientos al respecto [6].

Para los funcionarios y profesionales de los servicios de atención médica, se torna un desafío aproximarse a la comprensión de ideas y emociones de las personas de esta etnia. Al mapuche le afecta todo lo que ocurre en la naturaleza; cuando de alguna forma se rompe el equilibrio, las relaciones entre los seres se ven afectadas. Si alguien transgrede las leyes de la naturaleza, sufre las consecuencias del desequilibrio que ha provocado la enfermedad, tanto física como espiritual, conocida como kutran, kuxan, es decir, pérdida de salud, donde se experimentan dolores o perturbaciones desde el momento en que se introduce la enfermedad en su cuerpo [7], que puede ser Mapuche kuxan (propias de la cultura y vida mapuche) o wigka kuxan (con origen en la colonización y contacto con los wigka, huinca, persona de raza blanca) [8].

Junto al grupo anterior existen aquellas afecciones de origen divino o wепи тари (tierras de arriba), graves y difíciles de curar. Su comienzo siempre se debe a una transgresión de los designios de las deidades, ya sea de las/ os machis (curanderos, consejeros) o por no cumplir con los rituales pertinentes. También está la afección konün, la cual es causada por estar en contacto con la muerte en período de vulnerabilidad, como ocurre en las puérperas o lactantes, al hallarse cerca de un animal o persona agónica. Por último están las weza küxan o malas enfermedades, como weküfútun (provocada por un weküfe, espíritu dañino de la magia negra mapuche), kalkutun (generada por acción de la brujería) y perimontun (enfermedad sobrenatural producida por el poder de la naturaleza) [8].

En relación con las creencias y prácticas de los indígenas de El Paramito (Estado Mérida, Venezuela), al referirse a las enfermedades las consideran como "algo natural, que pueden venir de Dios, y algunas veces se producen por males que les echan o mojanazos". Expresan la importancia del equilibrio hombre-ambiente y reconocen elementos vitales como agua, tierra y aire, y las fuentes potenciales de patologías al ser contaminados dichos elementos. Los problemas de salud se relacionan más con violaciones a los preceptos morales que establece la Iglesia católica, es decir: "el cáncer les da a las mujeres que han tenido una vida promiscua o por faltar a sus deberes", y las de tipo crónico degenerativas, como las cardiovasculares. La hipertensión arterial (HTA) y el infarto son asociados directamente con los estilos de vida y los hábitos preponderantes, como son alcoholismo, tabaquismo y mascar chimó (tabaco curado), así como trastornos metabólicos del tipo de la diabetes y por gastropatías asociadas al tipo de alimentación [9].

En Colombia, las representaciones sociales de los indígenas embera chamí sobre el sistema médico tradicional y enfermedades foráneas se fundamentan en la Pacha Mama y los constructos de cosmogonía, cosmovisión y teoría de los tres mundos. La Pacha Mama es la encargada de brindar, por medio de las plantas y los elementos de la naturaleza, el saber a los facultativos para que pueda dar la sanación; en este sentido, solo actúa como intermediario entre la madre naturaleza y el paciente. Las enfermedades foráneas son desequilibrios o alteraciones físicobiológicas, propias de la "raza blanca", cuya presentación obedece a procesos de mestizaje, donde el indígena adopta costumbres del mundo occidental, principalmente en el componente alimentario. Dentro de algunas de las patologías, se rescatan las de tipo cardiovascular, debidas a la pérdida de identidad. Al igual que en los usuarios mapuches, para este pueblo el proceso 
salud-enfermedad es la armonía en las dimensiones psíquica, espiritual, social, ambiental y físicobiológica [10].

Volviendo a los mapuches, para lograr la sanación, un mapuche debe pasar por la conversación o negociación con la enfermedad, en la cual se recuerda su origen, para encontrar la causa del desequilibrio, que no es precisamente el organismo de la persona, por lo que se debe solicitar que retorne y que no provoque más daño en el afectado. El acto terapéutico es de competencia pública, además de ser una actividad sociorreligiosa y una obligación moral del grupo con el enfermo. Este proceso está ritualizado y se encamina a restablecer la armonía, "reconquistando" al cuerpo afectado de la influencia del mal, y a liberar el espacio comunitario de la presencia de factores perniciosos para el bienestar familiar y comunitario [8].

Los mapuches recurren a todos los sistemas médicos a su alcance, escogiendo aquellos que pueden tener mayor eficacia para resolver sus aflicciones, según la percepción que se tenga del origen de la enfermedad. "Estar bien" tiene, inclusive, una fuerte carga política, ya que se relaciona con los anhelos de emancipación y superación de la pobreza, y también al hecho de tener capacidad de pensar, discernir, consolidando el sentimiento de libertad y felicidad, para lo que ellos denominan ser persona o che [8].

El principal agente de salud reconocido y validado por la propia comunidad es el/la machi, mediador/a entre el mundo terrenal y el mundo espiritual, y su capacidad de desempeñar su rol está dado principalmente por el küpalme (historia de cada individuo, que es herencia de los antepasados) [11].

$\mathrm{La} /$ el machi representa la relación con el mundo sobrenatural, es la/el encargada/o de ejercer el papel de mejorar a los enfermos en un sentido integral, equilibrando una serie de fuerzas opuestas y complementarias que deben estar en armonía.

Otro agente médico, dentro de la cultura de este pueblo, son los Lawentuchufe o yerbateros, conocedor de las propiedades de las plantas, que utiliza para ayudar a recuperar la salud. A diferencia de la/el machi, no restablece el equilibrio espiritual [11].

En un estudio con población mapuche-williche, se obtuvo como resultado que frente al proceso saludenfermedad realizan un itinerario terapéutico que los lleva a complementar, alternar o sustituir la medicina occidental por la mapuche, para el tratamiento de la diabetes mellitus y la HTA, ya que las enfermedades cardiovasculares conceptualizadas por el modelo médico oficial-hegemónico no son parte de la matriz cultural mapuche-williche, constatándose dificultades en la adherencia al tratamiento impuesto por la biomedicina en usuarios que viven en territorios con alta concentración de población indígena [12].

A pesar de la Norma General Administrativa N..$^{\circ}$ 16, no se ha logrado una sincronización entre el agente de salud re- conocido por los mapuches, con el equipo de profesionales de los centros hospitalarios interculturales, evidenciado por una de las autoras en su rol de enfermera docente durante cuatro meses en el Hospital Makewe Pelale, región La Araucanía, donde se observó la falta de adherencia a las indicaciones y los tratamientos prescritos. Así mismo, los usuarios hipertensos adscritos al Programa de Salud Cardiovascular (PSCV), durante la hospitalización, presentan resistencia al tratamiento terapéutico, lo que se transforma en un problema grave, por corresponder a la principal causa de muerte en todo el mundo. En este establecimiento asistencial se atienden aproximadamente 6 mil personas, de las cuales el $80 \%$ pertenece al pueblo mapuche y los usuarios en el PSCV son 1776, y de estos, 311 personas son mayores de 65 años [13]. Cabe señalar que el Hospital Makewe es un establecimiento que se encuentra inserto en el territorio histórico Mapuche del centro sur de Chile, fundado en 1927 por los misioneros de la Iglesia anglicana, y recibe desde el año 1962 una subvención del Ministerio de Salud [13]. También es dable mencionar que, en Chile, el PSCV nace para contribuir a reducir la morbimortalidad asociada a las enfermedades cardiovasculares. Se crea en el año 2002 y a la fecha tiene más de 2 millones de personas inscritas. Ingresan personas del sistema público de salud, que tiene una o más de las siguientes patologías o factores: antecedente de una enfermedad cardiovascular ateroesclerótica documentada, diabetes mellitus, hipertensión arterial, dislipidemia y personas de 55 años y más que fuman [14].

Lo mismo sucede en el estudio realizado por Oliva y Narváez [15], cuyo objetivo era conocer los elementos socioculturales que influyen en los individuos al momento de no continuar con un tratamiento farmacológico en una enfermedad sintomática como lo es la HTA y describir las representaciones sociales pehuenches dentro de la lógica sanitaria cultural. En dicho estudio se destaca que el paciente indígena ve los fármacos como calmantes que no curan las causas de las enfermedades, debido a que estas se encuentran en un plano físico, espiritual y religioso, desde un punto de vista cultural, mágico y religioso, y no en contextos clínicos e individuales. Se concluye allí que los pehuenches no definen la HTA como una enfermedad válida e histórica; en consecuencia, para ellos, la farmacoterapia resulta suntuaria y disociativa de ritos y tradiciones que mantienen viva su cultura [15].

Otro estudio de los mismos autores, también ejecutado en el mismo tipo de población, demostró que la HTA, la diabetes y las dislipidemias no tienen un precedente cultural e histórico, sino más bien se oponen al sistema religioso; por esta razón, el tratamiento farmacológico no resulta efectivo, debido al escenario sociocultural particular [16].

Concretamente, respecto a la HTA, se calcula que en el año 2008 murieron 17,3 millones de personas, afectando por igual a ambos sexos, y más del $80 \%$ se producen en países de ingresos bajos y medios. Se estima que 
en 2030 morirán cerca de 23,3 millones de personas por enfermedades cardiovasculares, sobre todo por cardiopatías y accidente vascular cerebral [17].

Estudios epidemiológicos muestran que las tasas de enfermedades crónicas tienen una prevalencia mayor en comunidades indígenas de Chile, principalmente por los determinantes de salud asociados a las características culturales, a lo que se le suma su condición de ruralidad, que proporciona un mayor riesgo a estas patologías. La HTA, en lo particular, tiene tasas de prevalencia de $30,1 \%$, con un sostenido aumento anual [18], con $27 \%$ en población mapuche [19], y la existencia de problemas de cumplimiento farmacológico [15] y dietoterapéutico por parte de estos.

Con el propósito de contribuir a la calidad de vida del usuario mapuche, nace la inquietud de conocer su visión con respecto a la HTA, con la cual convive, el origen que da a esta, cómo afecta la realización de sus actividades diarias en zonas rurales y, por último, de qué manera acoge el tratamiento farmacológico, indicaciones del equipo de salud, de la herbolaria y de las/os machis, tomando en cuenta la familiaridad y los conocimientos heredados. Por lo anterior, surge la interrogante: ¿cuál es el significado que otorga a la HTA el usuario mapuche, adscrito al PSCV del Consultorio Makewe, en el año 2016? Cabe mencionar que este Consultorio está adscrito al Hospital Makewe Pelale.

Así, los objetivos de esta investigación se enfocan a: 1) explorar el significado de la hipertensión arterial; 2) descubrir lo que les ha significado, a los mapuches, vivir con HTA; 3 ) indagar por el tratamiento otorgado por el equipo de salud, y 4) develar tratamientos alternativos o complementarios.

\section{Metodología}

Dada la naturaleza del objeto de investigación, se optó por el paradigma cualitativo, ya que permite conocer la realidad desde una perspectiva de captar el significado particular que a cada hecho atribuye su propio protagonista, y de contemplar estos elementos como piezas de un conjunto sistemático, reconociendo que cada individuo es único en cuanto a personalidad, comportamiento, cultura, creencias, estilos de vida y visión que tiene del mundo que le rodea [20].

Se trabajó con base en la teoría fundamentada, que permite descubrir teorías que dormitan en los datos y que se sustentan en tres pilares fundamentales: método comparativo constante, muestreo teórico y codificación [21-23]. En este estudio se utiliza como un medio, ya que no se construirá una teoría formal, sino que sirve para comprender una realidad, pudiendo constituir un complemento para futuras investigaciones.
La muestra, no probabilística, intencionada de casos por criterio y por conveniencia [24], se conformó con veintidós sujetos cuyo criterio de inclusión fueron: adultos mayores (mayores de 65 años), que presenten capacidad oral, auditiva y cognitiva que permita una efectiva comunicación y comprensión del objetivo de estudio, con diagnóstico de HTA, tres años de permanencia en el PSCV, asistencia a todos los controles en el último año y aceptación de participar en forma voluntaria, con firma previa del consentimiento informado.

Para la recolección de datos se utilizó la técnica cualitativa de grupos focales (dos de siete integrantes cada uno) y ocho entrevistas en profundidad, recopilando los datos hasta llegar al punto de saturación, es decir, por repetición de ideas, hasta reunir pruebas y evidencias suficientes para garantizar la credibilidad de la investigación [24]. Como apoyo para recoger toda la información de manera exacta, se empleó grabadora de audio y notas de campo, con la correspondiente autorización.

En ambas técnicas participaron tres personas: una para registrar las notas de campo, otra para grabar la información, y la investigadora principal, quien orientó la técnica, comenzando la actividad con el enunciado de la pregunta orientadora y sirviéndose de un "guion" para recoger los temas a tratar. Las sesiones, de 45 minutos de duración, se realizaron en la Ruka, adosada al Hospital Makewe, que es un espacio reducido con un fogón, donde se dispusieron sillas para facilitar la participación de los informantes.

Teóricamente, el análisis de datos en este diseño se caracterizó por ser dinámico y creativo, basado en dos estrategias fundamentales: método comparativo constante y muestreo teórico. En lo concreto, se optó por el primero, ya que posterior a la recolección de datos se realizó codificación y análisis simultáneo [25].

Para la codificación se reconocieron cuatro tipos: 1) abierta: consistió en separar, detallar, comparar y conceptualizar los datos; 2) axial: donde se relacionaron códigos (categorías y propiedades) unos con otros, a través de ambos pensamientos, inductivo y deductivo; 3) teórica: proceso que permitió establecer una visión integradora de los testimonios que se habían fragmentado, producto de la codificación abierta; y 4) selectiva: fue el proceso en la elección de un símbolo como variable central [23]. La recolección y el análisis fueron concurrentes.

La confiabilidad se garantizó mediante el uso de los criterios de rigor, determinado por: valor de verdad o credibilidad, aplicabilidad o transferibilidad, consistencia o dependencia, y neutralidad o confirmabilidad [26].

Las consideraciones éticas estuvieron resguardadas desde el momento que fue un proyecto autorizado por el Comité Ético Científico de la Universidad de La Frontera, Chile (Acta 12 del 1. ${ }^{\circ}$ de enero de 2016), trabajándose con valor social: queda implícito que los resultados 
permitirán probables beneficios sociales y científicos; validez científica: mediante un diseño riguroso, cuyos productos, además, fueron triangulados por investigadores; selección equitativa: que resguardó la identificación de los informantes claves; proporción favorable de riesgo-beneficio: se cumplió respetando los principios de no-maleficencia y beneficencia, sin conflicto alguno para los participantes; evaluación independiente: hace referencia a la no distorsión de la investigación, producto de los intereses de los autores; consentimiento informado: incluyó respeto a una participación voluntaria y consciente, ofreciéndoles la oportunidad de plantear preguntas, dudas y retirarse del estudio si lo deseaban; y respeto a los inscritos: mediante la posibilidad de cambiar de opinión, asegurándoles confidencialidad de los datos y entrega de los resultados del estudio [27-29].

\section{Resultados}

En la medida en que se realizó la categorización y la codificación, se fue generando una síntesis de los resultados extraídos (véase tabla 1) del análisis de las entrevistas y grupos focales. En la tabla se observa la agrupación de cuatro categorías.

Para el primer objetivo, "Explorar el significado de la hipertensión arterial", se encontró la categoría "Significado de la hipertensión arterial", que fue organizada a partir del significado otorgado a la HTA por parte de los entrevistados, y subcategorizada en la "Definición del

Tabla 1. Categorías relacionadas con la hipertensión arterial.

\begin{tabular}{lll}
\hline \multicolumn{1}{c}{$\begin{array}{c}\text { Tema } \\
\text { central }\end{array}$} & Categoría & \multicolumn{1}{c}{ Subcategoría } \\
\hline Hipertensión & \multicolumn{1}{c}{ Significado } & Definición del concepto \\
\cline { 2 - 3 } & \multicolumn{1}{c}{ Causa } & Percepción \\
\cline { 2 - 3 } & Convivir & Cambios \\
\cline { 2 - 3 } & & $\begin{array}{l}\text { Farmacológico } \\
\text { Indicaciones del equipo de } \\
\text { Tratamiento }\end{array}$ \\
& $\begin{array}{l}\text { Salud } \\
\text { Herbolario } \\
\end{array}$ & Machi \\
\hline
\end{tabular}

concepto". Entre las asociaciones realizadas se devela como una enfermedad peligrosa, que implica patologías graves, y con signos y síntomas característicos; sin embargo, no identifican lo que ocurre en su organismo.

A continuación se señalan las citas más relevantes que reafirman lo anterior:

[...] es una enfermedad peligrosa, porque la presión, lo puede manejar a la persona entera, la enfermedad manda $[\ldots]$.
[...] la presión es muy delicado; la otra vez, yo no sé qué fue lo que comí o me hizo mal, que me dio parálisis, toda la mitad del cuerpo [...].

[...] era la presión de alta; de ahí comencé a controlarme yo, y por eso hasta ahora ando controlándome. Imagínese, no me había... controlándome; a lo mejor hubiera pasado mucho más cosas, más grave, porque me dijo la doctora: primero era un aviso, la segunda vez ya queda mal uno [...].

[...] yo creo, yo diría que es un poco grave para las personas $[\ldots]$.

[...] a mí me dio la vez pasa, me marié, se me daba vuelta todo $[\ldots]$.

[...] puede sufrir, le puede dar desmayo; de repente, yo me mareo y pienso que voy a caerme, como que asusta al corazón $[\ldots]$.

un vecino tenía esa presión alto, hizo sacarse toda la ropa, desnudarse... Le dio indignación [...].

Cabe señalar que la categoría "Causa de la hipertensión arterial", con su subcategoría "Percepción", no fue inicialmente objetivo del estudio; sin embargo, emerge en los discursos, y evidencia que la principal causa de esta patología crónica se debe al componente alimenticio. Aunque también relatan otros aspectos, entre ellos:

[...] puede ser por la sal [...].

$[\ldots]$ por las comidas, puede ser también [...].

[...] sabemos que la diabetes viene por lo dulce; entonces, la presión es la sal [...].

[...] sí, tiene que ser de la sal... por algo prohíben [...].

[...] ají poco también, porque empieza a marear la cabeza... todo lo picante y salado [...].

[...] las frituras también, y por eso dicen que hay que comer harta ensaladas, verduras y estar tomando los remedios, porque le dice ahí y entonces a uno se le quitan los mareos [...].

[...] puede ser colesterol, la grasa, estrictamente prohibido la carne de cordero... y vacuno [...].

[...] los condimentos...por que los condimentos de repente son muy fuertes y esos alteran los procesos de la sangre $[\ldots]$.

[...] de repente igual, cuando uno tiene una preocupación inconscientemente [...].

[...] puede ser la edad, mucho trabajo [...].

[...] sí, po uno se pregunta solo por qué [...] a mí me dijeron no más que tenía la presión alta, no más, claro, tiene la presión alta, y tome remedio pa' esto, uno pa'esto y uno pa'esto [...]. 
[...] puede dar por rabias puede ser, cansancio, trabajo $[\ldots]$.

$[\ldots]$ Estrés $[\ldots]$.

$[\ldots]$ mucho pensamiento $[\ldots]$.

$[\ldots]$ pasar rabia, malos ratos $[\ldots]$.

[...] el sistema nervioso, la soledad [...].

[...] es que nosotros no sabemos eso, no somos médicos para evaluar eso, ¿qué es eso lo que tiene que saber por qué es hipertensión arterial? [...].

[...] sí, bueno, es cuando la presión de la sangre, eehh sé lo que es más o menos, el médico me ha hecho saber, cuando... ¡aah, no sé explicarlo! Lo tengo claro, pero no sé cómo explicarlo, es por la presión sanguínea [...].

Con respecto al objetivo "Descubrir lo que le ha significado vivir con hipertensión arterial", emerge la categoría "Convivir con hipertensión arterial", y la subcategoría "Cambios", la que fue organizada a partir de las experiencias de los entrevistados, ya sea en su diario vivir, en el aspecto laboral, alimentario, emocional, y cómo esto ha significado modificar, de alguna manera, su estilo de vida.

A continuación se presentan algunos discursos representativos:

[...] me dio fuerte; yo me daba cuenta que hablaba, pero el chico no me entendía; hablaba raro, toda la mitad del cuerpo, $[. .$.$] la doctora: primero era un aviso,$ la segunda vez ya queda mal uno, vuelve mal, aunque vuelva, vuelve mal... yo empecé a caminar en el pasi1lo, fuerte, harto pensando en la enfermedad... y era la presión alta y ahí empezó a controlarme [...].

[...] pienso que voy a caerme, como que asusta al corazón $[\ldots . .$.$] .$

[...] le puede dar trombosis, problemas al corazón [...].

$[\ldots]$ hasta se puede morir $[\ldots]$.

$[\ldots]$ hasta le puede dar un infarto a la persona $[\ldots]$.

[...] hay gente que no puede trabajar mucho, y agacharse mucho $[\ldots]$.

[...] pero cuando trabaja mucho, cuando se cansa, la calor, la cabeza, buuu, apurado, se la puede... o sea, es más rápido cuando ve las estrellas [...].

[...] seguir controlándose no más, seguir tomando remedio, porque ahí queda bien uno; si no toma, anda mal. Si te dice el médico: "hay que tomar una en la mañana, dos o tres veces al día”, hay que hacerlo, hasta que anda bien; ahí andamos bien; si no andamos mal $[\ldots]$.

[...] hay dificultad también por tener la presión alta; tener mucho trabajo o mucha preocupación, se le acumula la preocupación en la cabeza y ni siquiera puede dormir, hasta puede volverse loco también [...]
[...] se van mermando las fuerzas, ya no tiene fuerza uno y se cansa uno. Eso comenzó con la presión; ahora ya no puedo mover una cosa pesadas [...].

[...] cuando estoy en altura [trabajando] se me da vuelta todo $[\ldots]$.

[...] yo trabajo en mi huerta y no tengo que agacharme mucho, poquito no más... Me empiezo a marear [...].

En relación con los objetivos "Indagar el tratamiento otorgado por el equipo de salud" y "Develar tratamientos alternativos o complementarios", cabe señalar que este último emergió como una subcategoría del tratamiento otorgado por el equipo de salud, con cuatro subcategorías, las que se describen a continuación.

- Categoría "Tratamiento de la hipertensión arterial": se aprecian variados manejos para mantener bajo control su patología crónica, agrupándolas en cuatro subcategorías:

1. Subcategoría "Fármacos": señalan los indicados por el médico y reconocen la entrega gratuita; sin embargo, expresan confusión en relación con los horarios, las condiciones, los nombres específicos y las acciones terapéuticas para cada uno de ellos, lo que se aprecia en las siguientes aseveraciones:

[...] Bueno, remedios le dan aquí... Todos los meses venimos a buscar $[\ldots]$.

[...] a mí me dan aspirina para el corazón, atenelol [atenolol], la bentiazina [benzodiacepina] y mmm... ¿cómo se llama el otro remedio? Son cuatro remedios y eso los tomo, dice, para el cuerpo [...].

[...] el enalapril y el paracetamol me dan, y una apirina chiquitito, eso que me dan para el corazón, y eso no más no me dan mucho $[\ldots]$.

[...] depende de cómo lo tome uno, porque hay gente que ya en caso de apuro pone tres, cuatro tabletas... eso no está bien [...].

[...] otros dicen que primero hay que comer, después tomarse la tableta, porque queda la tabletita entera en el estómago, según lo médico [...].

[...] no, no antes, después de tomar la tableta, hay que dejar pasar media hora [...].

[...] para que el remedio tenga ese tiempo de... pa deshacerse, porque luego, si come uno, no tiene lugar [...].

[...] tranquilizarse y se toma los medicamentos que le dan... tomo enalapril, aspirina, que me indicó el doctor $[\ldots]$.

[...] los remedios, y yo ando con la maquinita para tomar la presión [...].

[...] yo lo único que tomo más, más le tengo fe a los remedios, tomo el paracetamol, eso es lo que más... de hierbas no se ná [...]. 
2. Subcategoría "Indicaciones del equipo de salud": mayoritariamente, los discursos se enfocan a la alimentación:

[...] bueno, tenemos que cuidarnos, con lo que dice el médico, alimento, menos sal [...].

[...] y menos sal, no comer fritura [...].

[...] y no fumar también [...].

[...] no sé si ajo [...].

$[\ldots]$ comida ligerita $[\ldots]$.

[...] comida sana $[\ldots]$.

$[\ldots]$ no engordar mucho $[\ldots]$.

[...] y el mate debe ser bien lavaito, o sea, no amargo $[\ldots]$.

$[\ldots]$ no tiene que tomar café, $[\ldots]$, bebida tampoco tiene que ser, la más mala [...].

[...] a mí me exigen movimiento, si es posible, en la mañana correr un poco, hacer ejercicio [...].

[...] líquido, harto líquido [...].

[...] [seguimiento pauta nutricional] nooo, a veces no más, cuando tengo, cuando compramo, ahí teni$\operatorname{mos}[\ldots]$.

[...] son muy caro lo que le recetan [...].

[...] uno no puede consumir la sopa [instantánea con condimentos incorporados], a mí, me di cuenta sola, el doctor no me dijo na'.

A mí el médico, el doctor, me prohibió el caldo [alimento en conserva] [...].

Hay que comer ensalada, harta ensala', harta lechuga, harto repollo [...].

[...] me dijo el doctor que había que botar las primeras aguas y tomar un par de mates, no mucho [...].

- Subcategoría "Herbolaria": reconocen consumir algunas hierbas cuando asocian sus síntomas a un alza de presión; sin embargo, son cautelosos en su uso:

[...] hay hierbas que hacen bien; sí hay, pero hay que saber cuál es [...].

[...] nada de hierba; tiene que saber para qué es la hierba, para tomarla [...].

[...]el otro día yo tenía 16 , chupé un limón no más, cosas amargas, igual que el natre [arbusto perenne de 1 a $2 \mathrm{~m}$ de altura de color verde muy oscuro, antipirético $\mathrm{y}$ antiinflamatorio] [...].

[...] la alcachofa igual, como es amarga, entonces hace que baje la presión [...].

[...] solo cuando uno siente que la presión está un poco alta, porque lo amargo igual debilita [...].
[...] cochayuyo un poquito [alga marina comestible, de color marrón y tallo en forma de cinta, muy indicada en determinados tratamientos homeopáticos]; igual, dejarlo en limón, el día antes [...].

[...] que cuando me carga mucho, mucho, mucho, tomo agua de hierba de toronjil cuyano [...].

[...] tomo el tratamiento y al mate le echo un palito de jengibre... yo no siento ni un síntoma de que me sube la presión o no, si me canso, si me agito; ahí tomo romero con limón, una agüita [...].

[...] ah, las hierbas son mejores que todo: yo era del colesterol alto, y entonces el mismo médico Jaime Ibacache, que estaba aquí, y él me dijo: "tome hierbas" [...].

[...] igual el peumo [árbol siempre verde; la corteza y hojas se usan en lavativas para enfermedades del hígado], también es para la presión igual, limpia el estómago, la boca del estómago [...].

- Subcategoría "Machi": para la HTA, como para otras enfermedades, los informantes claves no reconocen a la/el machi e incluso algunos expresan su temor o rechazo, lo que se evidencia en las siguientes citas:

[...] yo vengo acá no más, ni a la machi tampoco [...].

[...] nunca me he atendido aquí con machi, nunca, y llevo hartos años atendiéndome acá [...].

[...] es peligroso ir al machi; por una parte, una vez casi me mató una machi, por los remedios, porque me dio remedios muy fuertes, por eso yo le tengo temor... acudí a la iglesia y el Señor me sanó ahí [...].

[...] yo le tengo harta fe (a la machi), gracias a Dios, sí, porque estaba mal... cuando va a la machi y no va con fe... si va así no más, no le hacen bien los remedios; sí, todas las cosas hay que hacerlas con fe [...].

[...] no creo en la machi tampoco. Fui a la machi porque realmente me sentía mal, pero resulta que fui a la otra machi de Nueva Imperial del Hospital [Establecimiento intercultural de mediana complejidad que brinda atención primaria ambulatoria y cerrada a la comuna de Nueva Imperial, y una atención secundaria abierta y cerrada para esta comuna y su área de influencia; se encuentra a 35,5 km de distancia del Consultorio Makewe], y también me dijo que tenía problemas uterinos y que me quedaban seis meses de vida; llevo cuatro años [...].

[...] ventajas y desventajas [atenderse con machi]: cuando fui cabro [Niño pequeño o en estado de pubertad] acudí a la machi y me sano; después no acudí más y sería todo [...].

[...] por la presión alta, nunca. Yo creo que rebota en los médicos; para la presión hay que venir a médico [...]. 


\section{Discusión}

En relación con el primer objetivo, "Explorar el significado de la hipertensión arterial", los entrevistados replican la información otorgada en la atención de salud, asociándola como una enfermedad importante y sumamente peligrosa, con una serie de complicaciones graves. Para ello utilizan nomenclatura de la salud occidental, como "parálisis" y "trombosis", semejante al estudio de Bermedo sobre los mapuche-williche, quienes conceptualizan las enfermedades cardiovasculares según el modelo médico oficial [12]. Sin embargo, al respecto, los resultados de esta investigación no coinciden con investigaciones nacionales efectuadas en la población pehuenche [16], ni con el trabajo realizado con indígenas de El Paramito [10].

Los informantes vinculan la HTA con "estrés", "pasar rabias", "malos ratos", "sistema nervioso", "soledad", "mucho pensamiento" y "consumo de sal y grasa", asimilándose en ello con un estudio venezolano, donde la población indígena relaciona la HTA y el infarto con los estilos de vida y hábitos como el alcoholismo, el tabaquismo y mascar chimó [10].

A su vez, solo uno de los entrevistados afirma que la HTA puede estar relacionada con algún grupo sanguíneo o asociada a debilidad, a diferencia de la investigación realizada por Oliva y Narváez en Colombia, donde se describe que son "desequilibrios o alteraciones físico biológicas, propias de la 'raza blanca', cuya presentación en los indígenas obedece a procesos de mestizaje" [15].

Se concluye que los informantes claves no logran definir esta patología claramente, mencionando que tienen noción de lo que es, sin lograr explicarla con sus propias palabras, asociándola a signos y síntomas junto a posibles causas. Es relacionada como una enfermedad compleja, con riesgos graves asociados.

Con respecto al segundo objetivo, "Descubrir lo que le ha significado vivir con hipertensión arterial", es un tema poco abordado por las investigaciones. Se rescata que la prevalencia de la HTA es baja cuando la alimentación está basada en comida sin procesar y con mínimos de pesticida, plaguicida u hormonas [12]. En el estudio presente, los informantes declaran que aunque tienen huertos para la producción de hortalizas para consumo propio, por sus bajos ingresos prefieren adquirirlos en el mercado urbano. Al respecto, sería importante que el incentivo del Gobierno para los huertos familiares se extienda a todo el territorio de La Araucanía.

En relación con el tercer objetivo: "Indagar el tratamiento otorgado por el equipo de salud", los participantes destacan que confían en la efectividad del tratamiento, concediendo, por ejemplo, al paracetamol, incluso eficacia en patologías no relacionadas a su efecto terapéutico: evidencian mejora en los síntomas asociados a la HTA. Sin embargo, existe confusión, debido a que el usuario presenta más de una patología, lo que podría implicar no reconocer los medicamentos específicos para la HTA. Lo develado se contrapone al estudio en población pehuenche, cuyos autores mencionan que las "tradiciones de estos usuarios no definen a la hipertensión arterial como una enfermedad válida e histórica, en consecuencia, la farmacoterapia resulta suntuaria y disociativa de ritos y tradiciones que mantienen viva su cultura" [16]. En la presente investigación, los informantes claves logran nombrar los medicamentos utilizados, sin tener completa claridad para qué es cada uno de ellos; evidencian mejora en los síntomas asociados a la HTA, sin gasto para ellos, permitiendo la validación al tratamiento.

Respecto a la alimentación sugerida por el equipo de salud, mencionan que no pueden cumplirla, debido al alto costo, y que se debe comprar en el pueblo más cercano. Como la mayoría son adultos mayores, son escasos los que poseen cultivos propios y crianza de animales para su autosustento, ya que ello requiere tiempo, dedicación e inversión económica que no disponen.

Finalmente, en el objetivo "Develar tratamientos alternativos o complementarios", los informantes claves, si bien expresan la utilización de herbolaria, información que obtienen por herencia, por recomendación de conocidos, por experiencias propias o por medicina complementaria, reconocen escasamente su utilidad; por esta misma razón, no consultan al Lawentuchufe y temen el uso de dichas hierbas, debido a la acción potente de algunas. De ahí la importancia de proporcionar educación sobre herbolaria y terapias complementarias, en la que se tome en cuenta su cosmovisión.

Un grupo limitado de entrevistados acuden activamente a la machi del Hospital de Imperial, debido a que en el Consultorio Makewe no tienen esta atención. Mencionan que no confían en la machi, debido a experiencias negativas a lo largo de la vida. También expresan que aquellas machis que son reconocidas por sus capacidades curativas, tienen un costo de atención muy elevado, lo que provoca que se distancien de este tipo de tratamiento. En otras situaciones se menciona que existe acercamiento a la religión católica de oriente, la cual les ha traído confort espiritual, lo que la medicina occidental no puede solucionar. Estos resultados se diferencian de los del estudio realizado por Bermedo con la población mapuche-williche, donde se menciona que "los usuarios en un itinerario terapéutico los lleva a complementar, alternar o sustituir la medicina occidental por la medicina mapuche para el tratamiento de la diabetes mellitus e hipertensión arterial" [12].

Finalmente, se puede concluir que el ámbito de la interculturalidad es un tema que con el paso de los años ha sido descuidado, pero no solo por las mismas instituciones que poseen este carácter, sino 
también por las políticas nacionales y los lineamientos ministeriales, ya que dentro de la búsqueda bibliográfica realizada no se halla alguna descripción respecto a la labor que cumple cada uno de los integrantes del equipo de salud. Si se refiere al PSCV, en el caso de la HTA en la población mapuche no hay una directriz para orientar el manejo y el tratamiento respetando su diversidad cultural; solo existe una indicación oficial y es entregada por medio de la Norma General Administrativa N. ${ }^{\circ}$ 16, ante la cual los usuarios tienen solo una alternativa: adaptarse a las terapias, y no viceversa. Existen capacitaciones internas que han sido brindadas por los mismos dirigentes pertenecientes a la comunidad en Makewe; a esto se agrega que muchos de los funcionarios pertenecen a esta misma etnia, por lo que se les facilita la comunicación con ellos; sin embargo, al momento de indicar una terapia, se encuentra la dificultad de que en el Consultorio Makewe está la de tipo farmacológica, ignorando cualquiera de origen natural.

Es importante tener en cuenta que los resultados de este estudio "Usuario mapuche: significado hipertensión arterial, Temuco, 2016", no son generalizables; solo representan la opinión de los pacientes mapuches con HTA, adscritos al PSCV del Consultorio Makewe, desde enero a diciembre del 2016.

\section{Financiamiento}

Investigación autofinanciada por los autores.

\section{Conflicto de intereses}

Ninguno declarado por los autores.

\section{Referencias}

1 Hasen-Narváez FN. Interculturalidad en salud: competencias en prácticas de salud con población indígena. Cienc. Enferm [internet]. 2012 [citado 2017 jul. 27]; 18(3):17-24. Disponible en: http://www.scielo.cl/scielo.php?script=sci_arttext\&pid $=$ S0717-95532012000300003

2 Zamudio T. Interculturalidad en salud en Chile: de la teoría a la práctica. Historia de los bio derechos y del pensamiento bioético [internet]. 2012 [citado 2017 sep. 11]. Disponible en: http://www. bioetica.org/cuadernos/contenidos/SAEZ.HTM

3 Programa Mapuche [internet]. 2012 [citado 2018 ago. 13]. Disponible en: https://www.araucanianorte.cl/index.php/programas/ programa-mapuche

4 Chile, Ministerio de Salud. Historia Hospital Makewe [internet]. 2009 [citado 2017 sep. 11]. Disponible en: http://www.bibliotecaminsal.cl/wp/wp-content/uploads/2011/09/Historia-HospitalMakewe.pdf

5 Alarcón A, Nahuelcheo Y. Creencias sobre el embarazo, parto y puerperio en la mujer mapuche: conversaciones privadas. Chungara [internet]. 2008 [citado 2017 sep. 12]; 40(2):193-202. Dis- ponible en: http://www.chungara.cl/Vols/2008/Vol40-2/Creencias_sobre_embarazo.pdf

6 Quezada A, Lolas F, Martin D. Prioridades en salud y salud intercultural. Estudio Bioética Social Univ. de Chile [internet]. 2007 [citado 2017 sep. 11]; (1):163-79. Disponible en: http://www.libros.uchile.cl/files/presses/1/monographs/252/submission/proof/ files/assets/basic-html/index.html\#page1

7 Díaz A, Pérez M, González C, Simón J. Conceptos de enfermedad y sanación en la cosmovisión mapuche e impacto de la cultura occidental. Cienc. Enferm [internet]. 2004 [citado 2017 sep. 17]; 10(1):9-16. Disponible en: http://www.scielo.cl/pdf/ cienf/v10n1/art02.pdf

8 Antona J. Los derechos humanos de los pueblos indígenas: El Az Mapu y el Caso Mapuche. Chile: Ciudad; 2014.

9 Pereira C, Valero F. La comunidad indígena de El Paramito: creencias y prácticas en torno a la salud y la enfermedad. Rev. Vzlana. de Soc. y Ant. [internet]. 2009 [citado 2016 ago. 27]; 19(56):495-517. Disponible en: http://www.redalyc.org/articulo. oa?id=70517761005

10 Cardona J, Rivera Y. Representaciones sociales sobre medicina tradicional y enfermedades foráneas en indígenas Embera Chamí de Colombia. Rev. Cubana Salud Pública. [internet]. 2012 [citado 2017 ago. 27]; 38(3):471-83. Disponible en: http://www.redalyc. org/articulo.oa? id=21423096013

11 Errázuriz G. El pueblo mapuche: historia, medicina y proyectos de coexistencia en el área de la salud (Primera parte). Rev. Chil. Pediatr. [internet]. 2006 [citado 2017 ago. 27]; 77(3):2904. Disponible en http://www.scielo.cl/scielo.php?pid=S037041062006000300010\&script=sci arttext

12 Bermedo S. Dimensiones y significados que adquiere el proceso salud/enfermedad/atención en usuarios mapuche-williche con diabetes mellitus e hipertensión arterial. Rev. Chil. Salud Pública. [internet]. 2015 [citado 2016 ago. 27]; 19(1):47-52. Disponible en: http://www.revistasaludpublica.uchile.cl/index.php/RCSP/article/viewFile/36339/37987

13 Chile, Biblioteca Ministerio de Salud "Dr. Bogoslav Juricic Turina". Historial Hospital Makewe [internet]. 2009. [citado 2018 ago. 13]. Disponible en: http://www.bibliotecaminsal.cl/wp/wpcontent/uploads/2011/09/Historia-Hospital-Makewe.pdf

14 Programa de Salud en Chile. Programa de Salud Cardiovascular [internet]. 2016. [citado 2018 ago. 13]. Disponible en: http://programasdesalud-chile.blogspot.com/2016/05/programa-de-saludcardiovascular.html

15 Oliva P, Narváez C. Representaciones sociales de salud y adherencia farmacológica antihipertensiva en población pehuenche. Rev. Chil. Salud Pública. [internet]. 2009 [citado 2016 nov. 24]; 13(2):67-71. Disponible en: http://www.revistasaludpublica.uchile.cl/index.php/RCSP/article/viewFile/593/498

16 Oliva P, Narváez C. Cumplimiento de tratamientos farmacológicos: una aproximación cualitativa a la realidad pehuenche. Medwave [internet]. 2013 [citado 2016 nov. 24]; 13(6). Disponible en: https://www.medwave.cl/link.cgi/Medwave/Estudios/Investigacion $/ 5742$

17 Organización Mundial de la Salud (OMS). Enfermedades cardiovasculares [internet]. Nota descriptiva 2013 [citado 2016 nov. 23]. Disponible en: http://www.who.int/mediacentre/factsheets/fs317/es/

18 Oliva P, Buhrinf K. Problemas de adherencia a dietoterapia en pacientes hipertensos pehuenches. Rev. Chil. Nutr. [internet]. 2011 [citado 2016 nov. 23]; 38(3):285-9. Disponible en: http://www. scielo.cl/pdf/rchnut/v38n3/art04.pdf

19 Carrasco E, Pérez F, Ángel B, Albala C, Santos L, Larenas G et al. Prevalencia de diabetes tipo 2 y obesidad en dos poblaciones 
aborígenes de Chile en ambiente urbano. Rev. Méd. Chil. [internet] 2004 [citado 2016 nov. 11]; 132(10):1189-97. Disponible en: http:// www.scielo.cl/pdf/rchnut/v38n3/art04.pdf

20 Ruiz J. Metodología de la investigación cualitativa. 4. ${ }^{\mathrm{a}}$ ed. Bilbao: Ed. Univ. de Deusto [internet]. 2009 [citado 2016 nov. 24]. Disponible en: http://www.iiicab.org.bo/Docs/doctorado/dip3version/ M2-3raV-DrErichar/investigacion-cualitativa.pdf

21 Cuesta $\mathrm{C}$ de la. Estrategias cualitativas más usadas en el campo de la salud. Nure investigación [internet]. 2006 [citado 2017 abril 9]. (25):1-4. Disponible en: https://rua.ua.es/dspace/bitstream/10045/17054/3/FMetod_25.pdf

22 Giraldo M. Abordaje de la investigación cualitativa a través de la teoría fundamentada en los datos. Ingeniería Industrial. Actualidad y Nuevas Tendencias. [internet]. 2011 [citado 2016 dic. 10]; 2(6):79-86. Disponible en: http://www.redalyc.org/articulo. oa? id=215021914006

23 Glaser B, Anselm L. The discovery of grounded theory: Strategies for qualitative research. New Brunswick (USA) and London (UK): Transaction publishers; 2009.
24 Polit D, Hungler B. Investigación científica en ciencias de la salud. 6. ${ }^{a}$ ed. México: McGraw-Hill Interamericana; 2000.

25 Rodríguez G, Gil J, García E. Metodología de la investigación cualitativa. Málaga: Aljibe; 1999.

26 Guba E, Lincoln Y. Effective evaluation: Improving the usefulness of evaluation results through responsive and naturalistic approaches. San Francisco: Jossey-Bass; 1981.

27 Burns N, Grove S. Investigación en Enfermería. Madrid: Elsevier; 2004

87 Botto A. Dimensión ética de la investigación cualitativa. Psiqui. Univ. [internet]. 2011 [citado 2017 abr. 18]; (4):354-7. Disponible en: http://revistagpu.cl/2011/GPU_Dic_2011_PDF/Editorial.pdf

29 Lolas F, Quezada A. Pautas éticas de investigación en sujetos humanos: nuevas perspectivas. Santiago de Chile: Programa Regional de Bioética OPS/OMS; 2003 